\title{
Impact of Admission Diagnosis on the Smoking Cessation Rate: A Brief Report From a Multi-centre Inpatient Smoking Cessation Programme in Singapore
}

\author{
Jason Jia Hao See', Kay Choong See ${ }^{2}$ \\ ${ }^{1}$ Department of Cardiology, Changi General Hospital, Singapore; ${ }^{2}$ Division of Respiratory and Critical Care Medicine, Department of Medicine, \\ National University Hospital, Singapore
}

Objectives: Few studies have been published regarding the relevance of the admission diagnosis to the smoking cessation rate. We studied smoking cessation rates in relation to admission diagnoses in our inpatient smoking cessation programmes.

Methods: This retrospective study included all patients recruited into our inpatient smoking cessation programmes at 2 institutions in Singapore between June 2008 and December 2016. Patients were given individualized intensive counselling and were followed up via phone interviews for up to 6-month to assess their smoking status. Multivariable logistic regression was used to analyse potential associations between admission diagnoses and 6-month abstinence.

Results: A total of 7194 patients were included in this study. The mean age was 54.1 years, and $93.2 \%$ were male. In total, 1778 patients (24.7\%) were abstinent at the 6-month follow-up call. Patients who quit smoking tended to be of Chinese ethnicity, have initiated smoking at a later age, be better educated, and have lower Fagerström Test of Nicotine Dependence scores. After adjusting for these factors, patients with a cardiovascular admission diagnosis had a significantly higher probability of quitting tobacco use than patients with a respiratory or other diagnosis.

Conclusions: In patients acutely admitted to the hospital, a diagnosis of cardiovascular disease was associated with the highest quit rate. Smoking cessation interventions need to be incorporated into all cardiovascular disease treatment pathways to leverage the patient's motivation and to improve the quit rate. In addition, patients in groups with lower quit rates may benefit from more intensive programmes to increase the rate of successful cessation.

Key words: Smoking cessation, Diagnosis, Cardiovascular disease, Singapore

\section{INTRODUCTION}

Received: April 16, 2020 Accepted: June 23, 2020

Corresponding author: Jason Jia Hao See, MBBS, MRCP Department of Cardiology, Changi General Hospital, 2 Simei Street 3, Singapore 529889, Singapore

E-mail: jason.see.j.h@singhealth.com.sg

This is an Open Access article distributed under the terms of the Creative Commons Attribution Non-Commercial License (https://creativecommons.org/licenses/bync/4.0/) which permits unrestricted non-commercial use, distribution, and reproduction in any medium, provided the original work is properly cited.
Tobacco use is a significant risk factor for chronic disease, in particular cardiovascular, respiratory, and cerebrovascular disease. Despite efforts at curbing this global epidemic, smoking remains one of the most preventable causes of death and kills more than 8 million people each year [1]. An admission to the hospital provides a good opportunity for smoking cessation, particularly when that admission is associated with the development of a new illness, which can often be a time of perceived 
vulnerability for the patient. Prior studies have shown relationships between disease development or morbidity and smoking cessation. However, these were largely general population studies that did not include formal smoking cessation interventions [2-4].

It is still unknown whether morbidity predicts smoking cessation outcomes in patients who undergo intensive smoking cessation counselling. A deeper understanding of this potential relationship can help better tailor smoking cessation efforts, especially if these efforts are incorporated into appropriate clinical management pathways that have been shown to improve care processes [5]. Furthermore, in patients with disease conditions that are not tobacco-related and that are associated with lower quit rates, additional interventions can be employed to motivate smoking cessation.

Our study therefore aimed to evaluate the impact of the admission diagnosis on the smoking cessation rate in patients who received smoking cessation counselling during their inpatient stay.

\section{METHODS}

\section{Study Design}

This retrospective study included all patients enrolled in our inpatient smoking cessation programmes at 2 hospitals in Singapore between June 2008 and December 2016. All patients admitted to the participating wards were screened to determine their smoking status, and those who consented to receive intensive smoking cessation counselling were enrolled in a programme. The study had no exclusion criteria.

\section{Details of the Inpatient Smoking Cessation Programmes}

The inpatient smoking cessation programmes in both hospitals were similar, with both programmes starting as partnerships between the hospitals and the Health Promotion Board (HPB) of Singapore with the aim of integrating tobacco cessation interventions into the existing clinical management of patients. Funding for manpower, training, and resources were provided by the HPB, with hospitals reporting back quarterly regarding reach and quit rates.

\section{Details of Counselling Sessions}

After the screening of admitted patients to determine their smoking status, identified smokers were provided with brief counselling. They were then offered to be enrolled into their hospital's inpatient smoking cessation programme, which included intensive counselling followed by phone interviews for up to 6 months. All patients who consented to enrolment into the programmes underwent intensive counselling, with each session lasting approximately 45 minutes. All counselling sessions were delivered by certified quit-smoking consultants [6] who had undergone standardized courses organized by the HPB. During these sessions, socio-demographic data and information regarding smoking patterns were collected. Fagerström Test of Nicotine Dependence (FTND) scores [7] were also obtained, and admission details were traced from medical records.

Admission diagnoses were further classified into 4 main groups (with predominant diagnoses in parentheses): cardiology (myocardial infarction, congestive cardiac failure, and angina), respiratory medicine (exacerbation of chronic obstructive pulmonary disease [COPD] or asthma and pneumonia), neurology (stroke and transient ischaemic attack), and others. Other diagnoses ranged widely, from orthopaedic conditions to minor infections, such as cellulitis.

Pharmacologic aids (various forms of nicotine replacement therapy as well as non-nicotine replacement medications such as bupropion and varenicline) were prescribed according to patients' preferences if not contraindicated. Patients were then followed up via phone interviews at 1 month, 3 months, and 6 months post-discharge for close monitoring of their smoking status and supportive contact.

\section{Outcomes}

The primary outcome was the self-reported abstinence rate at the time of the 6-month follow-up call. Only those who reported during the phone interview that they had not used cigarettes were considered to be successful quitters. Biochemical validation was not performed, per programme protocol. Patients who could not be contacted for follow-up were still included in the statistical analysis and were assumed to be non-quitters.

\section{Statistical Analysis}

Statistical analysis was performed to compare the characteristics of successful quitters to those of non-quitters and to determine whether the admission diagnosis had an impact on the quit rate. Data from both hospitals were combined to form a single set. Data were expressed as mean \pm standard devia- 
tion for continuous variables and as percentages or numbers for categorical variables. Categorical and dichotomous variables were compared using the chi-square test, while continuous variables were compared using the $t$-test. To adjust for potential confounders, the findings that were significant on univariable analysis were subjected to logistic regression modelling (via the enter method) to identify independent predictors for quitting and to estimate the odds ratios and $95 \%$ confidence intervals. The covariates of the model included age, age of smoking initiation, FTND score, race, admission classification, level of education, marital status, and the use of pharmacologic aids. All analyses were conducted using SPSS version 19 (IBM Corp., Armonk, NY, USA). All comparisons were 2-sided, and $p$-values $<0.05$ were considered to indicate statistical significance.

\section{Ethics Statement}

Ethical approval for this project was obtained from the respective review boards of both institutions (2017/2447 Singapore Health Services Centralised Institutional Review Board/ National Healthcare Group Domain-Specific Review Board).

\section{RESULTS}

A total of 7194 patients were included in the analysis. Their mean age was $54.1 \pm 14.0$ years, and $93.2 \%$ of the patients were male. Of the patients, 3563 (49.5\%) were Chinese, 2379 (33.1\%) Malay, 795 (11.1\%) Indian, and 457 (6.4\%) from other ethnic groups. As background information, the national demographics of Singapore are stable with regard to ethnic proportions, with approximately $74.4 \%$ Chinese, $13.4 \%$ Malay, $9.0 \%$ Indian, and 3.2\% other individuals as of June 2019 [8]. At the 6-month follow-up call, 1778 patients (24.7\%) reported abstinence from tobacco use.

Patients who quit smoking were more likely to be older, to have initiated smoking at a later age, to be married, to be better educated, and to have lower FTND scores. A small proportion of patients (8.8\%) were started on pharmacologic smoking cessation aids, but they had poorer quit rates (21.4 vs. $25.0 \%$ ) than patients not on pharmacotherapy $(p<0.05)$; however, this relationship was not found to be significant in the multivariable analysis $(p=0.939)$. Chinese patients had significantly higher quit rates than Malay or Indian patients $(p=0.017)$. Sex, having family members who smoked, and the number of previous quit attempts were not found to predict successful smok- ing cessation (Table 1).

Of the admissions, $45.8 \%(n=3292)$ were cardiology admissions, $22.1 \%(n=1589)$ were respiratory admissions, $15.3 \%$ $(n=1099)$ were neurology admissions, and $16.9 \%(n=1214)$ were other admissions. The quit rate was highest for cardiology admissions (28.0\%), followed by neurology $(25.9 \%)$, respiratory $(23.9 \%)$, and other admissions $(15.8 \%)(p<0.001)$.

Via multivariable analysis, the age of smoking initiation, FTND score, marital status, education level (a tertiary education compared to a primary education), and admission type (a cardiolo-

Table 1. Demographic predictors of smoking cessation at 6 months

\begin{tabular}{lccr}
\hline Variables & $\begin{array}{c}\text { Quit } \\
\text { smoking }\end{array}$ & $\begin{array}{c}\text { Continued } \\
\text { smoking }\end{array}$ & p-value \\
\hline Total & $1778(24.7)$ & $5416(75.3)$ & \\
Age (y) & $55.0 \pm 13.4$ & $53.8 \pm 14.2$ & 0.002 \\
Sex & & & 0.386 \\
Male & $1665(24.8)$ & $5038(75.2)$ & \\
Female & $113(23.0)$ & $378(77.0)$ & \\
Age of smoking initiation (y) & $18.4 \pm 6.7$ & $17.3 \pm 5.8$ & $<0.001$ \\
No. of previous quit attempts & $1.3 \pm 1.7$ & $1.3 \pm 1.7$ & 0.293 \\
FTND score & $3.8 \pm 2.4$ & $4.3 \pm 2.4$ & $<0.001$ \\
Ethnicity & & & 0.017 \\
Chinese & $935(26.2)$ & $2628(73.8)$ & \\
Malay & $556(23.4)$ & $1823(76.6)$ & \\
Indian & $174(21.9)$ & $621(78.1)$ & \\
Others & $113(24.7)$ & $344(75.3)$ & \\
Marital status & & & $<0.001$ \\
Single/divorced/widowed & $390(18.2)$ & $1752(81.8)$ & \\
Married & $1388(27.5)$ & $3664(72.5)$ & \\
Education & & & $<0.001$ \\
Primary & $136(21.4)$ & $500(78.6)$ & \\
Secondary & & & \\
Post-secondary or higher & $319(27.6)$ & $838(72.4)$ & \\
Admission classification & & & \\
Cardiology & $922(28.0)$ & $2370(72.0)$ & \\
Respiratory & $379(23.9)$ & $1210(76.1)$ & \\
Neurology & $285(25.9)$ & $814(74.1)$ & \\
Others & $192(15.8)$ & $1022(84.2)$ & \\
Family members who smoke & & & \\
Yes & & & \\
No & & & \\
Use of pharmacologic aids & & & \\
\hline No & & & \\
\hline
\end{tabular}

Values are presented as number (\%) or mean \pm standard deviation. FTND, Fagerström Test of Nicotine Dependence. 
Table 2. Logistic regression analysis of predictors of successful smoking cessation at 6-month follow-up

\begin{tabular}{|c|c|c|}
\hline Predictors & OR (95\% CI) & $p$-value \\
\hline Age (per year older) & $1.00(1.00,1.01)$ & 0.522 \\
\hline $\begin{array}{l}\text { Age of smoking initiation } \\
\text { (per year older) }\end{array}$ & $1.02(1.01,1.03)$ & $<0.001$ \\
\hline FTND score (per unit higher) $)^{1}$ & $0.91(0.89,0.93)$ & $<0.001$ \\
\hline \multicolumn{3}{|l|}{ Ethnicity } \\
\hline Chinese & 1.00 (reference) & \\
\hline Malay & $0.85(0.74,0.97)$ & 0.013 \\
\hline Indian & $0.72(0.59,0.89)$ & 0.002 \\
\hline Others & $0.85(0.67,1.08)$ & 0.187 \\
\hline Marital status & $1.62(1.40,1.86)$ & $<0.001$ \\
\hline \multicolumn{3}{|l|}{ Education level } \\
\hline Primary & $0.68(0.57,0.82)$ & $<0.001$ \\
\hline Secondary & $0.88(0.75,1.03)$ & 0.105 \\
\hline Post-secondary/tertiary & 1.00 (reference) & \\
\hline \multicolumn{3}{|l|}{ Admission classification } \\
\hline Cardiology & 1.00 (reference) & \\
\hline Respiratory & $0.80(0.68,0.93)$ & 0.005 \\
\hline Neurology & $0.90(0.76,1.06)$ & 0.196 \\
\hline Others & $0.49(0.40,0.59)$ & $<0.001$ \\
\hline Use of pharmacologic aids & $1.01(0.82,1.25)$ & 0.939 \\
\hline
\end{tabular}

$\mathrm{OR}$, odds ratio; $\mathrm{Cl}$, confidence interval; FTND, Fagerström Test of Nicotine Dependence.

${ }^{1} \mathrm{OR}<1.00$ suggests an inverse relationship.

gy admission compared to respiratory and other admissions) were found to be independent predictors of smoking cessation (Table 2).

\section{DISCUSSION}

Our smoking cessation programmes are focused on inpatient admissions to capitalize on the teachable moment when inpatient smokers are experiencing perceived vulnerability and thus may be more open to receiving help. These smokers may also find it easier to quit in an environment where smoking and the use of tobacco is prohibited. This perceived vulnerability may differ depending on the type of admission, in that a serious illness (such as a myocardial infarction or a stroke) may lead to a higher perceived susceptibility to disease, which then triggers changes in health behaviour. In a study by Newsom et al. [9] that examined data from the United States Health and Retirement Study, new diagnoses of chronic illnesses were associated with significant reductions in smoking prevalence. In that study, those diagnosed with heart disease, cancer, or stroke experienced the largest decrease. We sought to evaluate whether these differences would persist in patients enrolled in a smoking cessation programme.

In the present study, common predictors of smoking cessation, such as age of smoking initiation, marital status, FTND score, and educational levels, were identified $[10,11]$. With regards to admission diagnosis, cardiology, and neurology admissions were associated with the highest quit rates, with the frequent diagnoses under these 2 headings being myocardial infarction and stroke, respectively. A possible explanation for the high quit rates among these 2 groups of admission diagnoses could be that such illnesses were seen by patients as serious and potentially life-threatening, and the removal of smoking as a risk factor is known to lessen morbidity and facilitate recovery. Conversely, diagnoses other than cardiovascular, neurological, or respiratory were associated with a relatively low quit rate (15.8\%). This was likely due to lower disease severity or a lack of perceived association with smoking. Additionally, "other" diagnoses were not chronic illnesses and therefore did not serve as a constant reminder of the harms of smoking. Respiratory diagnoses were also associated with a significantly lower quit rate despite being smoking-related. Indeed, in a qualitative study by Eklund et al. [12], patients with COPD were found to have difficulty breaking their habit because of multiple factors and a lack of internal motivation, even though they had the knowledge about the harmful effects and consequences of continued smoking and COPD. Our findings highlight the need for the further study of strategies and techniques to improve the quit rates in these groups of patients. A study by Rigotti et al. [13] showed that sustained care interventions with automated telephone calls and free medication resulted in higher rates of post-discharge smoking cessation than standard care among hospitalized adults. Our programmes already utilize post-discharge follow-up telephone calls for supportive contact, but the take-up rate of pharmacologic smoking cessation aids was low in this study, which is an area that could be improved. Other potential additional strategies that have been shown in prior studies to result in higher quit rates could include the use of automated text message-based or social network-based interventions [14,15].

Interestingly, we found a trend (although statistically nonsignificant in the multivariable analysis) towards lower quit rates among patients who were administered pharmacologic aids. This is not in keeping with the published literature [16]. We are unable to explain this difference, but medication com- 
pliance and motivation to quit may have been relatively poor in the group that received these aids in the first place. We maintain that pharmacological aids should still be offered when indicated, per current smoking cessation guidance [17].

We also found that the quit rate among Chinese smokers was higher than that among Malay and Indian smokers. Malay and Indian individuals in our study population make up a disproportionate amount of smokers relative to the national demographics. Thus, this population of smokers may have greater exposure to friends and family who are fellow smokers, leading to mutual reinforcement of the smoking habit and lowering their quit rate.

The present study had a few limitations. First, as this was a retrospective study, our 2 institutions did not collect similar datasets, and we therefore analysed only variables that were common to the data of both institutions. Nevertheless, the variables included in our study have been demonstrated to be predictors of smoking cessation success, as shown in one of our authors' earlier publications [18], and we believe that our data were sufficient to evaluate for bias and show that admission diagnosis was an independent predictor of cessation success. Second, our study did not take into consideration specific differences in the management of the various admission diagnoses in our institutions. The implementation of clinical pathways for specific diagnoses such as myocardial infarction and COPD may have involved case management staff making additional phone calls or consults to the patient beyond those provided by our smoking cessation programmes and thereby reinforcing the message to quit smoking. Third, in a large proportion of the patients (10.5\%), data on education level were not collected. Nonetheless, the results from this study are in keeping with other studies that have shown a direct correspondence between quit rate and education level. Finally, in the present study, we did not carry out biochemical validation to verify abstinence. Although self-reporting is generally considered adequate in most smoking cessation studies given low rates of misreporting [19], a 2019 review by Benowitz et al. [20] showed that the biochemical verification of abstinence appears to be increasingly important, as social norms and other personal factors affect the accuracy of reporting of smoking behaviour. However, as our programmes relied on phone interviews to follow up with participants, it would not have been feasible to employ biochemical verification. We also had little reason to assume that untrue answers were differently distributed between those who successfully quit and those who did not.
In conclusion, we found significant differences in the smoking cessation rate based on admission diagnosis. We postulate that the most likely reason for this is the increased motivation to quit when a smoker is diagnosed with a high-morbidity disease, such as myocardial infarction (cardiovascular disease) or stroke (neurological disease), since the removal of smoking as a risk factor is known to reduce morbidity and facilitate recovery. For patients with diagnoses that were associated with lower quit rates, further efforts could be implemented to improve their cessation rates, such as the increased use of pharmacologic aids or a greater frequency of supportive contact, either in person or via the phone. Such measures are deserving of further study.

\section{CONFLICT OF INTEREST}

The authors have no conflicts of interest associated with the material presented in this paper.

\section{FUNDING}

None.

\section{ACKNOWLEDGEMENTS}

The authors would like to acknowledge the Health Promotion Board of Singapore for its continued support of our inpatient smoking cessation programmes. We would also like to thank Ms Tan Pei Ting from the Clinical Trials and Research Unit at Changi General Hospital for providing statistical consultation.

\section{AUTHOR CONTRIBUTIONS}

Conceptualization: JJHS. Data curation: JJHS, KCS. Formal analysis: JJHS, KCS. Funding acquisition: None. Methodology: JJHS, KCS. Writing - original draft: JJHS. Writing - review \& editing: JJHS, KCS.

\section{ORCID}

Jason Jia Hao See https://orcid.org/0000-0001-7647-0197

Kay Choong See https://orcid.org/0000-0003-2528-7282 


\section{REFERENCES}

1. World Health Organization. WHO report on the global tobacco epidemic 2019 [cited 2020 Mar 20]. Available from: https:// www.who.int/tobacco/global_report/en/.

2. Tsai AC, Lin YA, Tsai HJ. Predictors of smoking cessation in 5066-year-old male Taiwanese smokers: a 7-year national cohort study. Arch Gerontol Geriatr 2012;55(2):295-300.

3. Yang JJ, Song M, Yoon HS, Lee HW, Lee Y, Lee SA, et al. What are the major determinants in the success of smoking cessation: results from the health examinees study. PLoS One 2015; 10(12):e0143303.

4. Holm M, Schiöler L, Andersson E, Forsberg B, Gislason T, Janson C, et al. Predictors of smoking cessation: a longitudinal study in a large cohort of smokers. Respir Med 2017;132:164-169.

5. Cheah J. Clinical pathways--an evaluation of its impact on the quality of care in an acute care general hospital in Singapore. Singapore Med J 2000;41(7):335-346.

6. Health Promotion Board. Certification for quit smoking consultants; 2017 [cited 2018 Jun 1]. Available from: https://www. hpb.gov.sg/community/smoking-cessation-programme/certification-for-quit-smoking-consultants-(level-2).

7. Heatherton TF, Kozlowski LT, Frecker RC, Fagerström KO. The Fagerström test for nicotine dependence: a revision of the Fagerström Tolerance Questionnaire. Br J Addict 1991;86(9): 1119-1127.

8. Department of Statistics Singapore. Population dashboard; 2019 [cited 2020 May 31]. Available from: https://www.singstat.gov.sg/find-data/search-by-theme/population/population-and-population-structure/visualising-data/populationdashboard.

9. Newsom JT, Huguet N, McCarthy MJ, Ramage-Morin P, Kaplan MS, Bernier J, et al. Health behavior change following chronic illness in middle and later life. J Gerontol B Psychol Sci Soc Sci 2012;67(3):279-288.

10. Caponnetto P, Polosa R. Common predictors of smoking ces- sation in clinical practice. Respir Med 2008;102(8):1182-1192.

11. Gilman SE, Martin LT, Abrams DB, Kawachi I, Kubzansky L, Loucks EB, et al. Educational attainment and cigarette smoking: a causal association? Int J Epidemiol 2008;37(3):615-624.

12. Eklund BM, Nilsson S, Hedman L, Lindberg I. Why do smokers diagnosed with COPD not quit smoking?-a qualitative study. Tob Induced Dis 2012;10:17.

13. Rigotti NA, Regan S, Levy DE, Japuntich S, Chang Y, Park ER, et al. Sustained care intervention and postdischarge smoking cessation among hospitalized adults: a randomized clinical trial. JAMA 2014;312(7):719-728.

14. Whittaker R, McRobbie H, Bullen C, Rodgers A, Gu Y, Dobson R. Mobile phone text messaging and app-based interventions for smoking cessation. Cochrane Database Syst Rev 2019; 10(10):CD006611.

15. Pechmann C, Delucchi K, Lakon CM, Prochaska JJ. Randomised controlled trial evaluation of Tweet2Quit: a social network quit-smoking intervention. Tob Control 2017;26(2):188-194.

16. Cahill K, Stevens S, Perera R, Lancaster T. Pharmacological interventions for smoking cessation: an overview and network meta-analysis. Cochrane Database Syst Rev 2013;(5):CD009329.

17. Barua RS, Rigotti NA, Benowitz NL, Cummings KM, Jazayeri MA, Morris PB, et al. 2018 ACC expert consensus decision pathway on tobacco cessation treatment: a report of the American College of Cardiology Task Force on Clinical Expert Consensus Documents. J Am Coll Cardiol 2018;72(25):3332-3365.

18. See JH, Yong TH, Poh SL, Lum YC. Smoker motivations and predictors of smoking cessation: lessons from an inpatient smoking cessation programme. Singapore Med J 2019;60(11): 583-589.

19. SRNT Subcommittee on Biochemical Verification. Biochemical verification of tobacco use and cessation. Nicotine Tob Res 2002;4(2):149-159.

20. Benowitz NL, Bernert JT, Foulds J, Hecht SS, Jacob P, Jarvis MJ, et al. Biochemical verification of tobacco use and abstinence: 2019 update. Nicotine Tob Res 2020;22(7):1086-1097. 